\title{
Estenosis subglótica secundaria a granulomatosis de Wegener. Reporte de un caso y revisión de la literatura
}

\author{
Subglottic stenosis secondary to Wegener's granulomatosis. \\ A case report and literature review.
}

Christian Olavarría L1', Daniel Muñoz S².

\begin{abstract}
RESUMEN
La granulomatosis de Wegener es una enfermedad multisistémica de origen autoinmune con numerosas manifestaciones otorrinolaringológicas. Dentro de éstas destaca la estenosis subglótica que puede eventualmente comprometer la vida del paciente. Su diagnóstico requiere un alto grado de sospecha, pues se ha visto que su desarrollo es independiente de la actividad de la enfermedad sistémica y debe basarse principalmente en la visión endoscópica de la lesión. El tratamiento de la estenosis subglótica en pacientes con granulomatosis de Wegener es complejo, requiere a menudo de repetidas intervenciones debido a reestenosis. Actualmente, no existe consenso en la técnica quirúrgica más adecuada, por lo que la decisión final deberá basarse en la evaluación de cada caso en particular. Presentamos el caso de un paciente con estenosis subglótica y granulomatosis de Wegener atendido en nuestro servicio y revisamos la literatura con énfasis en las alternativas de tratamiento de esta enfermedad.
\end{abstract}

Palabras clave: Estenosis subglótica, granulomatosis de Wegener, vasculitis, tratamiento.

\begin{abstract}
Wegener's granulomatosis is a autoimmune systemic disease with numerous upper respiratory tract manifestations. Among these, subglottic stenosis represents a potentially lethal complication. Its diagnosis is complicated by the fact that it develops independently from the systemic disease, as it is mainly based on endoscopic examination. Treating subglottic stenosis in patients suffering from Wegener's granulomatosis is complex, it often requires repeated interventions due to restenosis. There is no current consensus on the most adequate surgical technique, and thus it must be evaluated and decided on a case-to-case basis. We present the case of a subglottic stenosis and Wegener's granulomatosis patient from our department. In addition, we reviewed the literature, emphasizing treatment alternatives.
\end{abstract}

Key words: Subglottic stenosis, Wegener's granulomatosis, vasculitis, treatment.

Médico, Servicio de Otorrinolaringología, Hospital Clínico Universidad de Chile.

Médico Cirujano, Universidad de Chile. 


\section{INTRODUCCIÓN}

La granulomatosis de Wegener (GW) es una enfermedad crónica sistémica de origen autoinmune. Fue descrita inicialmente por Klinger en $1932 \mathrm{y}$, en forma acabada, por Friedrich Wegener como una tríada de vasculitis granulomatosa de las vías respiratorias, vasculitis sistémica y gromeluronefritis focal necrotizante ${ }^{1,2}$. Actualmente es clasificada como una vasculitis de pequeño vaso asociada a anticuerpos citoplasmáticos antineutrófilos, especialmente subtipo c (c-ANCA), útiles para su diagnóstico precoz y seguimiento ${ }^{3}$.

Esta enfermedad tiene $82 \%$ de mortalidad a un año del inicio de los síntomas ${ }^{4}$. Se presenta a cualquier edad, pero es más frecuente entre la $5^{\mathrm{a}} \mathrm{y}$ $6^{a}$ década de vida, con predominio en el sexo masculino. En EE.UU., la prevalencia es de 3 casos en 100.000 habitantes, casi exclusivamente en caucásicos 5 .

La granulomatosis de Wegener presenta un amplio rango de manifestaciones otorrinolaringológicas, ya sea como complicación o como síntoma inicial. El compromiso en cabeza y cuello es la manifestación más frecuente seguida por el pulmonar, articular, ocular y renal ${ }^{6}$.

En las cavidades nasosinusales, puede manifestarse como sinusitis, epistaxis, perforación septal 0 deformidad nasal en silla de montar. Se ha descrito compromiso otológico en $25 \%-40 \%$ de los casos, dado principalmente por otitis media con efusión e hipoacusia sensorioneural secundaria a vasculitis coclear? ${ }^{7}$. En la Tabla 1 se resumen las manifestaciones de la GW de interés en otorrinolaringología.

En la laringe, la estenosis subglótica se ha reportado en el 9\%-23\% de los pacientes ${ }^{8}$ con GW, pudiendo coexistir más de una lesión en el árbol laringo-tráqueo-bronquial. Es más frecuente en mujeres y en aquellos pacientes con diagnóstico de GW a edad temprana9. Esta región es un área particularmente susceptible de estrechamiento debido a la presencia de un anillo cartilaginoso completo como es el cartílago cricoides $^{10}$. Se ha descrito también, que los pacientes con GW y estenosis subglótica, padecen mayor compromiso sinusal y menor compromiso renal y pulmonar ${ }^{11}$.

La presentación clínica puede ir desde casos asintomáticos hasta el compromiso vital por estrechamiento de la vía aérea. La disnea de esfuerzo es el síntoma más frecuente de la estenosis subglótica $(79 \%-82 \%)$ y puede acompañarse de cambios en la voz y disfonía, estridor 0 tos $^{12}$. La disfonía puede deberse a parálisis de cuerdas vocales, posiblemente debido a eventos vasculíticos sobre los nervios laríngeos ${ }^{13} 0$ a alteraciones de la articulación cricoaritenoidea.

Tabla 1. Manifestaciones otorrinolaringológicas de la granulomatosis de Wegener

Otológicas

Destrucción y deformidad de pabellón auricular

Disfunción tubaria

Otitis media con efusión

Otomastoiditis granulomatosa

Meningitis

Parálisis nervio facial

Hipoacusia

Vértigo

Nariz y senos paranasales

Rinosinusitis crónica

Epistaxis

Obstrucción nasal

Perforación septal

Rinorrea

Deformidad nasal (en silla de montar)

Costras nasales

Epífora
Cavidad oral y glándulas salivales

Estomatitis ulcerativa

Inflamación mucosa crónica

Fístula oro-antral

Necrosis de paladar

Nódulos mucosos

Hiperplasia gingival

Sialoadenitis

Parotidomegalia

Laringe

Estenosis subglótica

Estenosis supraglótica

Parálisis de cuerdas vocales 
La vía aérea requiere ser evaluada endoscópicamente (idealmente mediante traqueobroncoscopía) y la estenosis subglótica debe ser clasificada usando la escala de Cotton-Mayer, según porcentaje de obstrucción del diámetro de la vía aérea ${ }^{14}$ (Tabla 2).

El diagnóstico de la estenosis debe complementarse siempre con la medición de c-ANCA. Cabe señalar que inicialmente sólo un poco más de la mitad de los pacientes es positivo al momento del diagnóstico del compromiso subglótico, pudiendo el resto hacerse positivo con posterioridad $^{15}$. Asimismo, nos podemos apoyar en el estudio histológico a través de la presencia de vasculitis, granulomas necrotizantes y células gigantes². Las biopsias de lesiones subglóticas (realizadas en pacientes ANCA+ y con estenosis) son consistentes con una GW en el $5 \%-15 \%$ de los casos. Por el contrario, las biopsias nasales han mostrado $82 \%$ de sensibilidad para GW ${ }^{16}$.

Con respecto al diagnóstico por imágenes, la tomografía computada provee una buena aproximación para evaluar lesiones laringotraqueales. Además, tiene la ventaja de realizar reconstrucciones sagitales lo que confiere mayor precisión en definir los límites superior e inferior de la lesión y el compromiso de cuerdas vocales ${ }^{17}$.

El diagnóstico diferencial de la GW involucra una variedad de enfermedades granulomatosas y autoinmunes tales como la sarcoidosis, enfermedad de Churg-Strauss, poliarteritis nodosa, poliangeítis microscópica y policondritis recidivante ${ }^{2-5}$.
Tabla 2. Clasificación de la estenosis subglótica (Cotton - Myer) $^{14}$

\begin{tabular}{|lc|}
\hline Grado & Obstrucción (\%) \\
\hline I & $0-50$ \\
II & $51-70$ \\
III & $71-99$ \\
IV & 100 \\
\hline
\end{tabular}

\section{CASO CLÍNICO}

Paciente de sexo masculino de 42 años de edad, con antecedente de aspergilosis pulmonar resuelta con lobectomía izquierda, intubación endotraqueal y otitis media por efusión tratada. Refiere cuadro de 2 años de evolución con rinorrea serosa, obstrucción nasal, hipoacusia bilateral progresiva y disfonía de 1 año de evolución. Desde hace 6 meses se agrega tos irritativa y dificultad respiratoria progresiva. Destaca al examen físico la presencia de deformidad nasal en "silla de montar», estridor laríngeo bifásico leve y compromiso ocular a derecha tipo epiescleritis.

La nasofibroscopía muestra fosas nasales con abundantes costras, sinequias entre el tabique nasal y cornete medio. En la región glótica, se observan cuerdas vocales móviles con sinequia anterior que ocluye en al menos $50 \%$ el lumen y presencia de material costroso en laringe (Figura 1). La región

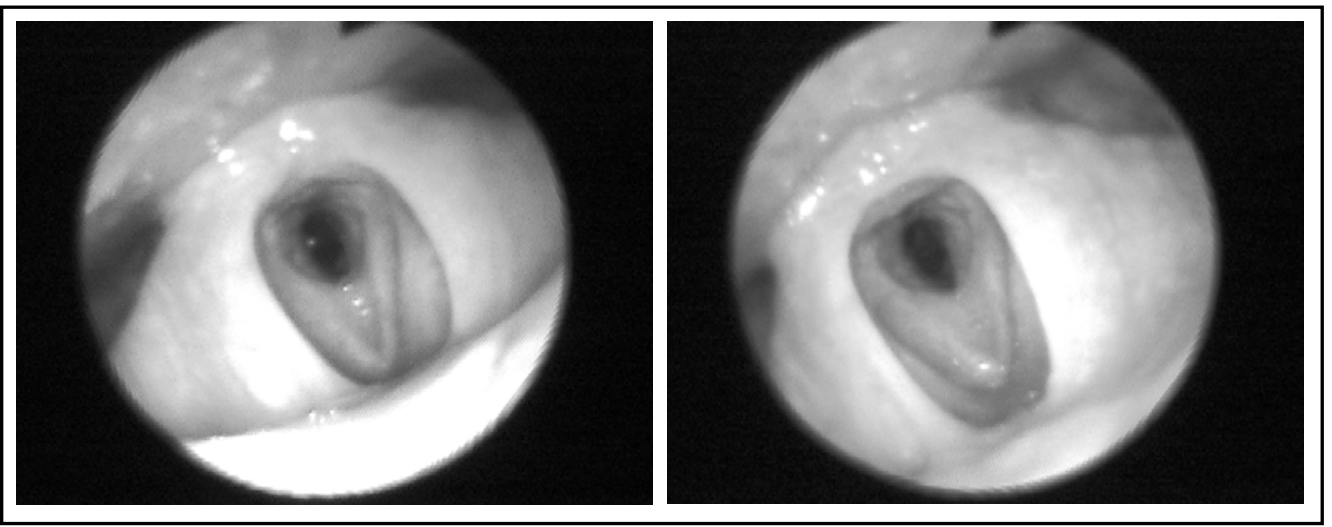

Figura 1. Imagen endoscópica de la estenosis subglótica del paciente en discusión. Destaca el estrechamiento de aproximadamente el $50 \%$ del lumen respiratorio. 
Revisión del tratamiento quirúrgico para estenosis subglótica en GW, publicado en la literatura durante los últimos 12 años

\begin{tabular}{|c|c|c|c|c|c|c|}
\hline Autor & Tratamiento & $\begin{array}{c}n^{\circ} \\
\text { pacientes }\end{array}$ & $\begin{array}{c}\text { Seguimiento } \\
(\overline{\mathrm{x}} \text { meses })\end{array}$ & $\begin{array}{l}\text { Procedimientos } \\
\qquad(\overline{\mathrm{x}})\end{array}$ & $\begin{array}{c}\text { ANCA + } \\
(\%)\end{array}$ & $\begin{array}{c}\text { Complicaciones } \\
(\%)\end{array}$ \\
\hline Lanford $^{19}(1996)$ & $\mathrm{DE}+\mathrm{Cl}$ & 20 & 35 & 3 & 100 & 0,08 \\
\hline Herridge ${ }^{33}$ (1996) & RLT & 3 & 85,5 & 1 & 100 & 0 \\
\hline Sttapaerts $^{31}(2000)$ & $\mathrm{DE}+\mathrm{Cl}$ & 1 & 6 & 1 & 100 & 0 \\
\hline Utzig $^{32}(2002)$ & $\mathrm{DE}+\mathrm{Cl}+$ escisión quirúrgica & 7 & 30 & 1,2 & 80 & 14 \\
\hline Watters ${ }^{28}(2003)$ & Stent de Nitinol & 1 & 48 & 1 & 100 & 0 \\
\hline Gluth"11 (2003) & DE + Láser CO2 & 12 & 76,8 & 2,3 & 100 & - \\
\hline Hoffman ${ }^{25}$ (2003) & $\mathrm{DE}+\mathrm{Cl}+$ escisión quirúrgica & 21 & 24 & 3,3 & - & 3 \\
\hline Shvero ${ }^{26}(2007)$ & Láser C02 o ND:YAG & 5 & 21,6 & 7,8 & 100 & 0 \\
\hline Shoeckkenbroek ${ }^{24}(2008)$ & $\mathrm{DE}$ & 9 & 25,4 & 2,4 & 100 & 0 \\
\hline
\end{tabular}

DE =Dilatación endoscópica; $\mathrm{Cl}$ =corticoides intralesionales; RLT =Reconstrucción laringotraqueal.

subglótica presenta un estrechamiento de su lumen en aproximadamente $50 \%$. La tomografía computada de cavidades paranasales evidencia extensa ocupación del laberinto etmoidal izquierdo, engrosamiento de mucosa maxilar derecha con extensa ocupación maxilar izquierda y ocupación parcial del seno esfenoidal izquierdo (Figura 2).

Se toma biopsia de tejido laríngeo (bajo laringoscopía directa) y biopsia de cavidad nasal (cornete medio izquierdo). El resultado del estudio histológico sólo mostró, para ambas muestras obtenidas, un proceso inflamatorio crónico. Desde el punto de vista inmunológico, destaca la negatividad de anticuerpos como c-ANCA, p-ANCA y serología de VIH (-). Dado el compromiso respiratorio progresivo, se decide su manejo con terapia inmunosupresora (corticoesteroides), con buena respuesta sintomática a corto plazo.

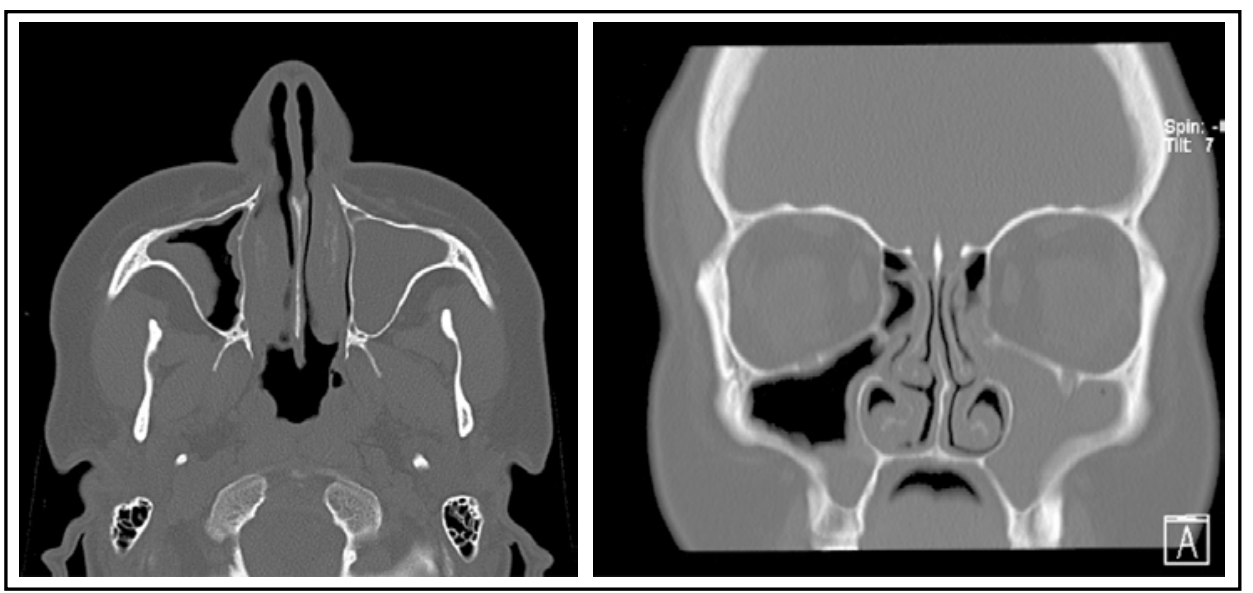

Figura 2. Tomografía computada de cavidades paranasales del paciente con granulomatosis de Wegener. Destaca el extenso compromiso, especialmente, del seno maxilar izquierdo. 


\section{DISCUSIÓN}

La estenosis subglótica ocurre en aproximadamente el $9 \%-23 \%$ de los pacientes con GW y representa la manifestación más frecuente en vía aérea ${ }^{8}$. Según Rottem y cols., es cinco veces más frecuente en pacientes cuyo diagnóstico de GW se hizo antes de los 19 años de edad'. El segmento estenótico puede estar limitado a la subglotis 0 extenderse hasta 3 ó $4 \mathrm{~cm}$ hacia la tráquea y eventualmente hacia la supraglotis ${ }^{18}$ y aproximadamente la mitad de los pacientes con compromiso laríngeo requerirán traqueostomía en algún momento de su evolución ${ }^{5}$.

La clínica de la estenosis subglótica es variable y puede ocurrir en ausencia de otras características de GW activa. Según Langford y cols., $79 \%$ de los casos presentan disnea, $61 \%$ disfonía y estridor y $22 \%$ tos $^{19}$. Resulta llamativo que algunos pacientes con GW con estenosis en estadios iniciales se han diagnosticado como asmáticos, pues los test de función pulmonar son anormales en cerca del $60 \%$ de los pacientes con estenosis subglótica, mostrando un patrón obstructivo que confunde al médico tratante ${ }^{34,35}$. Cabe señalar, que en nuestro caso existen otros antecedentes de factores predisponentes al desarrollo de estenosis subglótica, como la intubación endotraqueal, lo que sumado a GW claramente contribuye al proceso patológico.

Nuestro paciente no presentó alteraciones en su estudio serológico, como se hubiese esperado. Sin embargo, este hecho no excluye la enfermedad pues se ha reportado vasculitis de pequeño vaso ANCA negativo, estimándose que 1 de cada 5 pacientes con la enfermedad inicial pueden ser seronegativos ${ }^{20}$. Resulta de importancia entonces, el seguimiento de los títulos de ANCA, pues en algunos pacientes los títulos negativos pueden hacerse positivos con el tiempo. La decisión de iniciar tratamiento inmunosupresor en nuestro caso fue empírica, dada la progresión de la disnea y la consiguiente disminución de la capacidad funcional. La exclusión de otros diagnósticos diferenciales, se basó en la apreciación clínicosindromática de la granulomatosis de Wegener, que en este caso es la que más concordaba dentro del espectro de enfermedades granulomatosas.
El tratamiento de la estenosis subglótica en pacientes con GW es complejo y requiere a menudo de repetidas intervenciones, por una alta tasa de recurrencia de la estenosis ${ }^{21}$.

\section{Tratamiento médico}

Se basa en el uso de drogas inmunosupresoras como corticosteroides, ciclofosfamida, entre otras. Éstas, han sido útiles en la enfermedad sistémica, pero no así en estenosis subglótica, siendo posible incluso que ésta debute mientras el paciente está en tratamiento ${ }^{22}$. Su éxito en aliviar la obstrucción varía entre $22 \%$ y $26 \%{ }^{23}$.

\section{Tratamiento quirúrgico}

\section{- Traqueostomía}

La obstrucción severa de la vía aérea requiere traqueostomía, ya sea temporal o permanente, hasta en el $60 \%$ de los casos ${ }^{5}$. La decanulación del traqueostoma ha sido utilizada por algunos autores como un parámetro de éxito de otros tratamientos ${ }^{15}$.

- Tratamiento endoscópico

Los procedimientos endoscópicos han sido, en general, exitosos en el manejo de esta condición. La dilatación endoscópica es efectiva la mayoría de las veces para casos Cotton-Meyer grado I-II, con escasas complicaciones, pero son pocos los casos reportados ${ }^{24}$. Por otro lado, se ha usado la dilatación endoscópica, combinada con inyecciones de corticoides intralesionales. Hoffman y cols., reclutaron 21 pacientes que requirieron entre 2 ó 3 procedimientos al año para mantener la apertura de la vía aérea. Ninguno de ellos requirió una nueva traqueostomía y la tasa de complicaciones fue menor y sin secuelas a largo plazo ${ }^{25}$.

- Tratamiento láser

Para lesiones distales a las cuerdas vocales se ha usado láser Nd:YAG con $80 \%$ de éxito 0 decanulación durante el seguimiento. Sin embargo, todos los pacientes requirieron múltiples tratamientos ${ }^{26}$. Según otros autores, los procedimientos láser provocan fibrosis extensa y aquellos pacientes son más difíciles de manejar con posterioridad ${ }^{27}$. 
- Laringotraqueoplastía

Consiste en la reconstrucción laringotraqueal con injerto de cartílago costal. Gluth y cols, reportaron una serie de pacientes con excelentes resultados, pues la totalidad de ellos fueron decanulados y no hubo evidencia de falla del injerto ${ }^{11}$. Lebovics y cols realizaron laringotraqueoplastía en 5 pacientes que habían tenido múltiples procedimientos endoscópicos fallidos, logrando decanulación con adecuada calidad de voz en todos los casos ${ }^{16}$.

- Prótesis de vía aérea

Watters y cols., utilizaron un stent expandible de nitinol (aleación de níquel y titanio) en la vía aérea estenótica en un paciente que evolucionó sin complicaciones y sin tejido de granulación ${ }^{28}$. Sin embargo, su uso no está exento de complicaciones, por lo que no existe acuerdo en su recomendación ${ }^{29,30,34}$.

\section{CONCLUSIONES}

Resulta trascendental un alto grado de sospecha clínica en la GW dado que su pronóstico final depende, en gran medida, de un diagnóstico pre$\mathrm{coz}$.

El compromiso laringotraqueal, potencialmente mortal, debe evaluarse dirigidamente en todos estos pacientes, principalmente bajo visión endoscópica. Debemos recordar que, a pesar de la alta sensibilidad de c-ANCA, existe la posibilidad de enfermedad seronegativa. Así, como en nuestro caso, la decisión terapéutica no debiera retrasarse a la espera de un diagnóstico serológico pues ésta es una entidad que eventualmente puede comprometer la vida del paciente si no es tratada oportunamente.

La estrategia terapéutica debe ser evaluada caso a caso. El uso de traqueostomía, si hay compromiso respiratorio vital, es indiscutible. Por otro lado, de acuerdo a la evidencia, parece sensato el uso inicial de dilatación endoscópica idealmente con corticoides intralesionales. Si los procedimientos endoscópicos no son satisfactorios, debe evaluarse la reconstrucción laringotraqueal abierta. El uso de stents de vía aérea no está exento de complicaciones, por lo que su recomendación dependerá sólo de que el beneficio de usarlos sea superior al riesgo de complicarse.

\section{BIBLIOGRAFÍA}

1. Khan AM, Elahi F, Hashmi SR, Mahida KH, Ingrams DR. Wegener's granulomatosis: a rare, chronic and multisystem disease. Surgeon 2006; 4(1): 45-52.

2. ERICKSON VR, HWAng PH. Wegener's granulomatosis: current trends in diagnosis and management. Curr Opin Otolaryngol Head Neck Surg 2007; 15(3): 170-6.

3. Gaughan RK, De Santo LW, McDonald TJ. Use of anticytoplasmicautoantibodies in the diagnosis of Wegener's granulomatosis with subglotticstenosis. Laryngoscope 1990; 100(6): 561-3.

4. Thickett DR, Richter AG, Nathani N, Perkins GD, Harper L. Pulmonary manifestations of antineutrophilcytoplasmic antibody (ANCA)positive vasculitis. Rheumatology (Oxford) 2006; 45(3): 261-8.

5. Gottschlich S, Ambrosch P, Kramkowski D, Laudien M, Buchelt T, Gross WL, Hellmich B. Head and neck manifestations of Wegener's granulomatosis. Rhinology 2006; 44(4): 227-33.

6. Rasmussen N. Management of the ear, nose, and throat manifestations of Wegener granulomatosis: an otorhinolaryngologist's perspective. Curr Opin Rheumatol 2001; 13(1): 3-11.

7. Takagi D, Nakamaru $Y$, Maguchi $S$, Furuta $Y$, FUKUDA S. Otologic manifestations of Wegener's granulomatosis. Laryngoscope 2002; 112(9): 1684-90.

8. Seo P. Wegener's granulomatosis: managing more than inflammation. Curr Opin Rheumatol 2008; 20(1): 10-6.

9. Rottem M, Fauci as, Hallahan CW, Kerr GS, Lebovics R, Leavitt RY, Hoffman GS. Wegener granulomatosis in children and adolescents: clinical presentation and outcome. J Pediatr 1993; 122(1): 26-31.

10. LANGFORD CA. Wegener's granulomatosis: current and upcoming therapies. Arthritis Res Ther 2003; 5(4): 180-91. 
11. Gluth MB, Shinners PA, Kasperbauer JL. Subglotticstenosis associated with Wegener's granulomatosis. Laryngoscope 2003; 113(8): 1304-7.

12. Polychronopoulos VS, Prakash UB, Golbin JM, Edel ES, Specks U. Airway involvement in Wegener's granulomatosis. Rheum Dis Clin North Am 2007; 33(4): 755-75, vi.

13. Alahn $A$, Hogg RP, Drake lee $A$. Wegener's granulomatosis and subglotticstenosis: management of the airway. J Laryngol Otol 2004; 118(10): 786-90.

14. Blaivas AJ, Strauss W, Yudd M. Subglotticstenosis as a complication of Wegener's granulomatosis. Prim Care Respir J 2008; 17(2): 114-6.

15. Bandi V, Munnur U, Braman SS. Airway problems in patients with rheumatologic disorders. Crit Care Clin 2002; 18(4): 749-65.

16. Lebovics RS, Hoffman GS, Leavitt RY, Kerr GS, Travis WD, Kammerer W, Hallahan C, Rottem M, Faucl AS. The management of subglotticstenosis in patients with Wegener's granulomatosis. Laryngoscope 1992; $102(12$ Pt 1): 1341-5.

17. Allen SD, Harvey CJ. Imaging of Wegener's granulomatosis. $\mathrm{Br} J$ Radiol 2007; 80(957): 757-65.

18. Belloso A, Estrach C, Keith A0. Supraglotticstenosis in localized Wegener granulomatosis. Ear Nose Throat J 2008; 87(7): E11-4.

19. Langford CA, Sneller MC, Hallahan CW, Hoffman GS, Kammerer WA, Talar-Williams C, Fauci AS, LeBovics RS. Clinical features and therapeutic management of subglotticstenosis in patients with Wegener's granulomatosis. Arthritis Rheum 1996; 39(10): 1754-60.

20. Carrie $S$, Hughes KB, Watson MG. Negative ANCA in Wegener's granulomatosis. J Laryngol Otol 1994; 108(5): 420-2.

21. LORENZ RR. Adult laryngotrachealstenosis: etiology and surgical management. Curr Opin Otolaryngol Head Neck Surg 2003; 11(6): 467-72.

22. Thurnher D, Moukarbel RV, Novak CB, Gullane PJ. The glottis and subglottis: an otolaryngologist's perspective. Thorac Surg Clin 2007; 17(4): 549-60.
23. Loehrl TA, Smith TL. Inflammatory and granulomatous lesions of the larynx and pharynx. Am J Med 2001; 111 Suppl 8A: 113S-117S.

24. Schokkenbroek AA, Franssen CF, Dikkers FG. Dilatation tracheoscopy for laryngeal and tracheal stenosis in patients with Wegener's granulomatosis. Eur Arch Otorhinolaryngol 2008; 265(5): 549-55.

25. Hoffman GS, Thomas-Golbanow CK, Chan J, Akst LM, ELIACHAR I. Treatment of subglotticstenosis, due to Wegener's granulomatosis, with intralesional corticosteroids and dilation. $J$ Rheumatol 2003; 30(5): 1017-21.

26. Shvero J, Shitrit D, Koren R, Shalomi D, Kramer MR. Endoscopic laser surgery for subglotticstenosis in Wegener's granulomatosis. Yonsei Med J 2007; 48(5): 748-53.

27. Ahmad I, Lee WC, Nagendran V, Wilson F, SHORTRIDGE RT. Localised Wegener's granulomatosis in otolaryngology: a review of six cases. ORL J Otorhinolaryngol Relat Spec 2000; 62(3): 149-55.

28. Watters K, Russell J. Subglotticstenosis in Wegener's granulomatosis and the nitinolstent. Laryngoscope 2003; 113(12): 2222-4.

29. Eliachar I, Chan J, Aкst L. New approaches to the management of subglotticstenosis in Wegener's granulomatosis. Cleve Clin J Med 2002; 69 Suppl 2: SIl149-51.

30. Yumoto E, SaekI K, Kadota Y. Subglotticstenosis in Wegener's granulomatosis limited to the head and neck region. Ear Nose Throat J 1997; 76(8): 571-4.

31. Stappaerts I, Van Laer C, Deschepper K, Van de Heyning P, VermelRE P. Endoscopic management of severe subglotticstenosis in Wegener's granulomatosis. Clin Rheumatol 2000; 19(4): 315-7.

32. Utzig MJ, Warzelhan J, Wertzel H, Berwanger I, HASSE J. Role of thoracic surgery and interventional bronchoscopy in Wegener's granulomatosis. Ann Thorac Surg 2002; 74(6): 1948-52.

33. Herridge MS, Pearson FG, Downey GP. Subglotticstenosis complicating Wegener's granulomatosis: surgical repair as a viable treatment option. J Thorac Cardiovasc Surg 1996; 111(5): 961-6. 
34. Simon C, McCammon S, Quinn F. Subglottic Stenosis in Wegener Granulomatosis. Grand Rounds Presentation, UTMB, Dept. of Otolaryngology. 21 de mayo, 2001. <http:// www.utmb.edu/otoref/grnds/GrndsIndex.html> [Consulta, enero de 2009].
35. Solans-Laqué R, Bosch-Gil J, Canela $M$, Lorente J, Pallisa E, Vilardell-Tarrés M. Clinical features and therapeutic management of subglottic stenosis in patients with Wegener's granulomatosis. Lupus 2008; 17(9): 832-6. 